\title{
A GRIDDED ELECTRON GUN FOR A SHEET BEAM KLYSTRON
}

\author{
M. E. Read, G. Miram, and R.L. Ives, Calabazas Creek Research, Inc., Saratoga, CA, 95070-3753 \\ V. Ivanov and A. Krasnykh, Stanford Linear Accelerator Center, Menlo Park, CA 94025
}

\section{Abstract}

This paper describes the development of an electron gun for a sheet beam klystron. Initially intended for accelerator applications, the gun can operate at a higher perveance than one with a cylindrically symmetric beam. Results of 2D and 3D simulations are discussed.

Calabazas Creek Research, Inc. (CCR) is developing rectangular, gridded, thermionic, dispenser-cathode guns for sheet beam devices. The first application is expected to be klystrons for advanced particle accelerators and colliders.[1] The current generation of accelerators typically use klystrons with a cylindrical beam generated by a Pierce-type electron gun. As RF power is pushed to higher levels, space charge forces in the electron beam limit the amount of current that can be transmitted at a given voltage. The options are to increase the beam voltage, leading to problems with X-Ray shielding and modulator and power supply design, or to develop new techniques for lowering the space charge forces in the electron beam.

In this device, the beam has a rectangular cross section. The thickness is constrained as in a normal, cylindrically symmetric klystron with a Pierce gun; however, the width of the beam is many times the thickness. The resulting cross sectional area is much larger than in the conventional device. This allows much higher current and/or a lower voltage before space charge forces become too high.

The current program addresses issues related to beam formation at the emitter surface and implementation of shadow and control grids in a rectangular geometry. It is directed toward a robust, cost-effective, and reliable

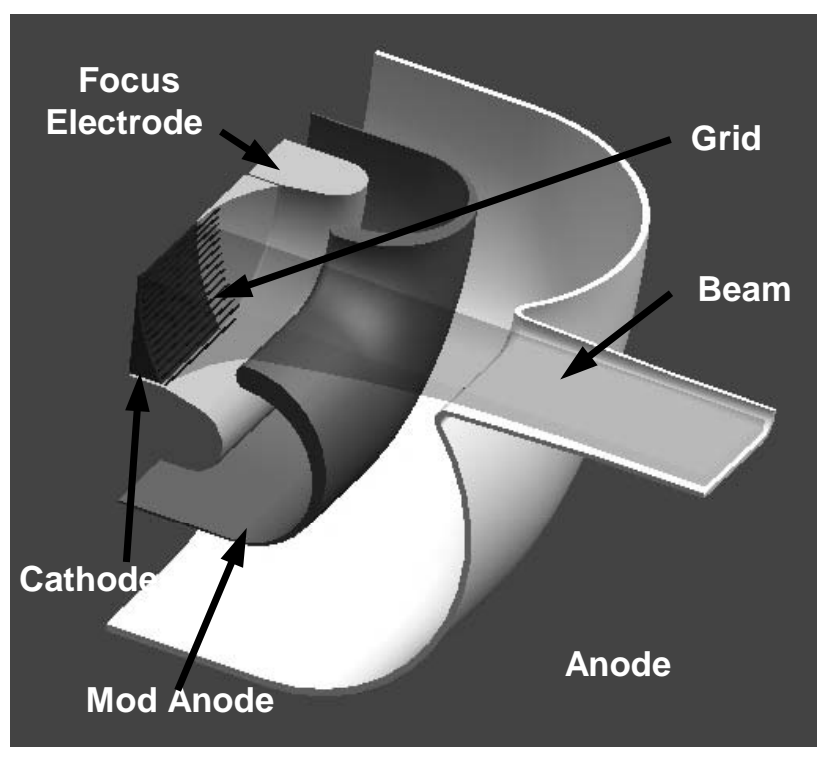

Figure 1. Geometry of the sheet beam gun. One quarter of the gun is shown, with the cathode on the left and the axes of symmetry toward the top and left. The mod anode is included to grade the field and minimize the possibility of an arc directly from the cathode to the anode.

mechanical design. A prototype device will be developed at $415 \mathrm{kV}, 250 \mathrm{~A}$ for a $40 \mathrm{MW}, \mathrm{X}$-Band, sheet-beam klystron. The cathode will have $100 \mathrm{~cm}^{2}$ of cathode area with an average cathode current loading of $2.5 \mathrm{~A} / \mathrm{cm}^{2}$. For short pulse formation, the use of a grid was chosen. The geometry of the gun is shown in Figure 1.

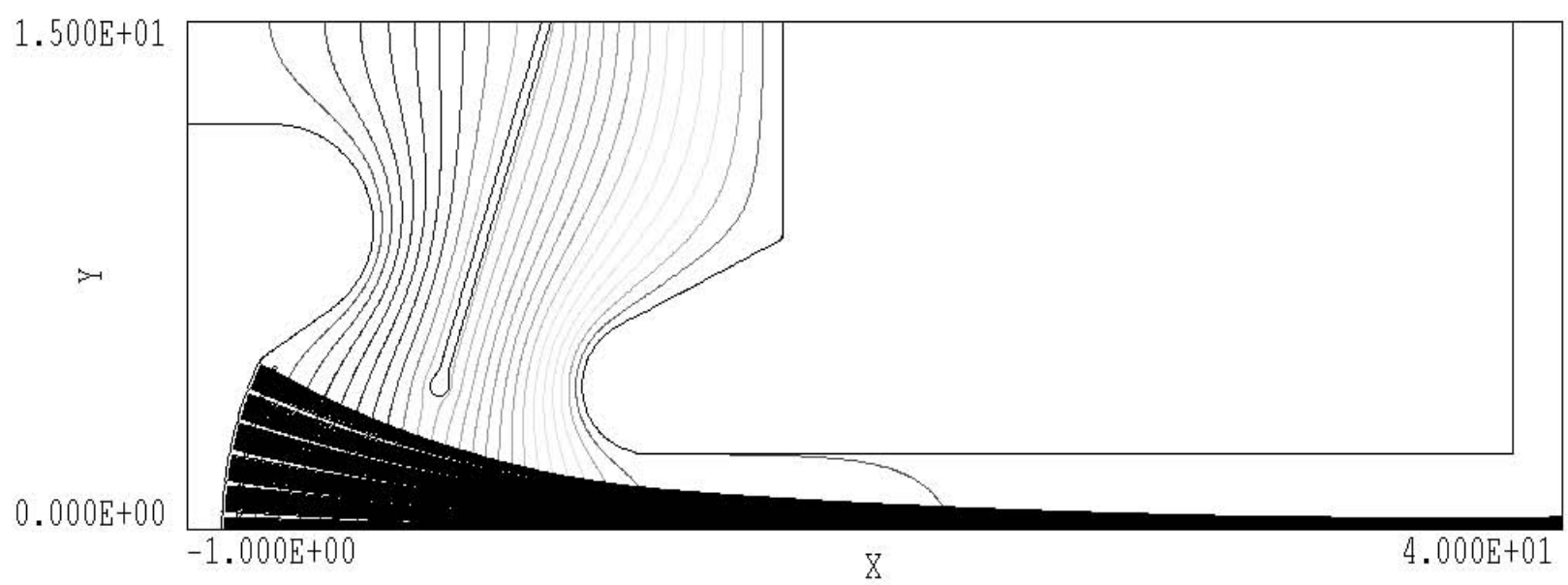

Figure 2. Trajectories from a 2D simulation using TRAK, including the shadow grid. Dimensions in $\mathrm{cm}$. 

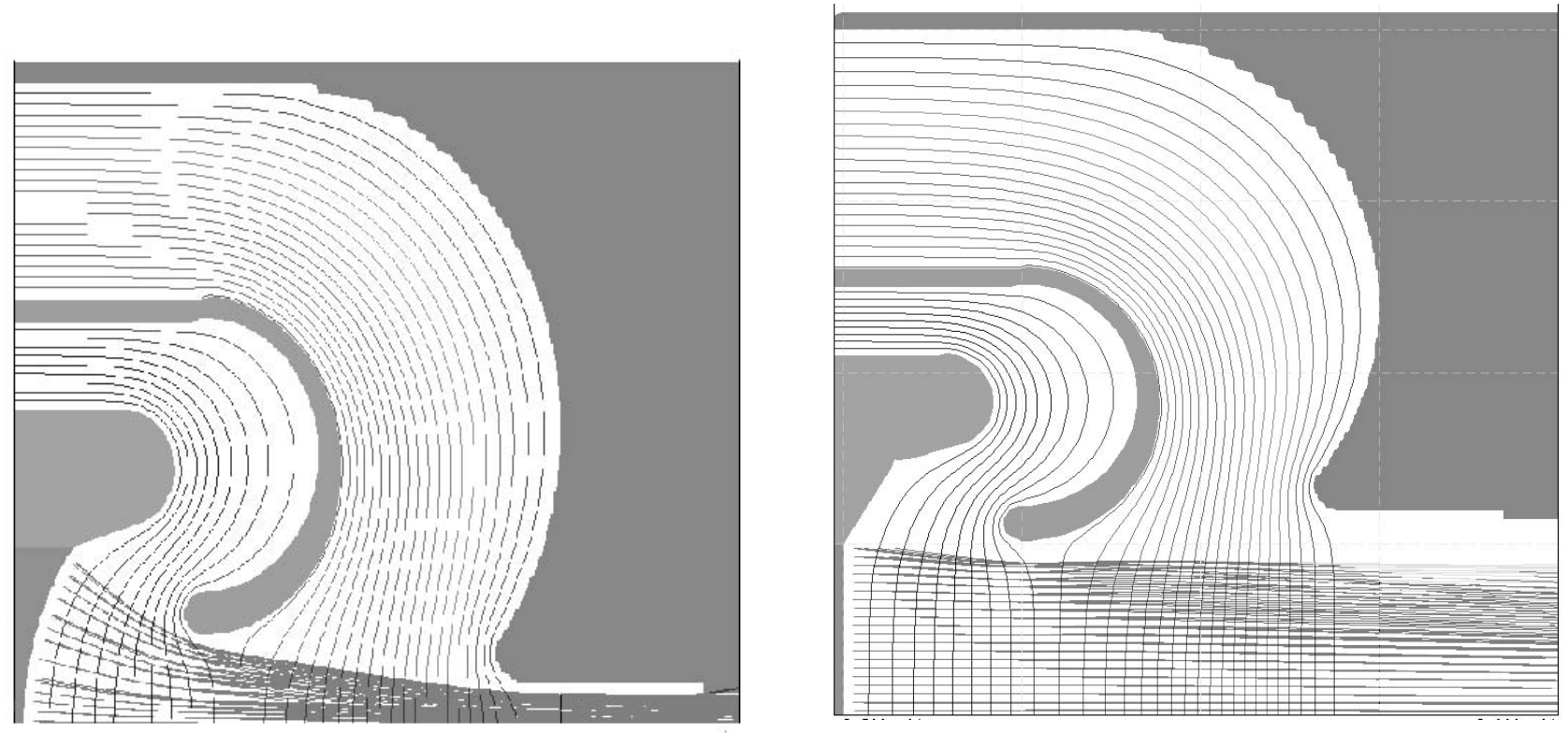

Figure 3. Trajectories as calculated using the 3D code OmniTrak. Cross sections near the axes of symmetry for two planes normal to the direction of propagation.

The gun is being designed with a combination of 2-D and 3-D codes. 2-D codes were used to determine the starting point for the electrodes to produce the compression (which is in only one direction.) These results showed that a very high quality beam could be achieved even in the presence of the shadow grid. A plot of the 2-D results is shown in Figure 2.

The 3-D design is done using the codes TOPAZ and OmniTrak.[2] Both codes include relativistic effects and self magnetic fields of the beam. OmniTrak uses a structured grid, while the grid in TOPAZ is unstructured. Examination of initial designs showed good agreement between the codes.

Initial results from OmiTrak are shown in Figure 3. The beam is compressed only in one dimension. Compression from the $8.4 \mathrm{~cm}$ high cathode to close to the desired $8 \mathrm{~mm}$ thickness is shown in the left. There is almost no compression in the other plane; however, in the plane of compression, trajectories originating close to the edges of the cathode are over compressed. This is shown in Figure 4. The additional compression occurs because electrons near the cathode edge see the effect of the focusing electrodes in both transverse planes. Efforts are continuing to reshape the corners of the focus electrodes to eliminate this effect.

Self-fields were found to be very important for this geometry. Figure 5 shows the trajectories in the plane of compression calculated without the self magnetic field. The compression is reduced to the point that a substantial interception of the beam by the anode. Clearly, designing the electrodes without consideration of the self-fields would result in over compression of the beam.

A DC power supply will drive the gun, with the grid being used to pulse the beam. While this approach allows sharper pulse formation than can be achieved with a cathode pulser and is potentially less expensive, it does

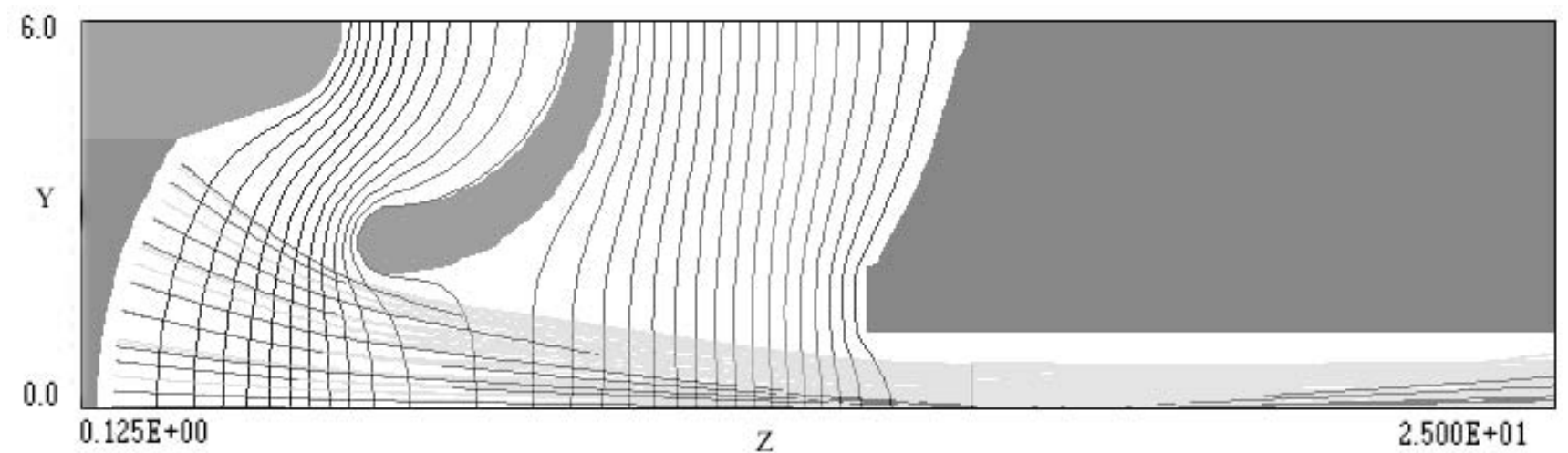

Figure 4. Trajectories (darker lines) near the "X" edges of the cathode. The solution space has been shrunk from that of Figure 1 by applying potentials determined from the electrostatic solutions of Figure 1 to the upper boundary of this solution. 


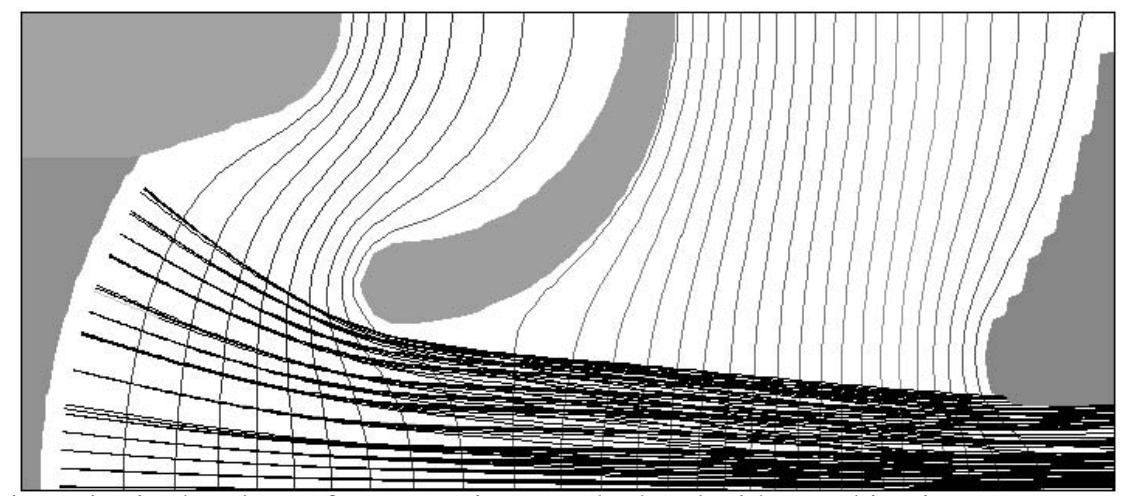

Figure 5. Trajectories in the plane of compression as calculated without taking into account self magnetic fields.

place stricter limits on the field gradients, both in vacuum and in the oil of the high voltage tank. The design goal is to limit the vacuum fields to less than $75 \mathrm{kV} / \mathrm{cm}$ on the beam formation electrodes and $40 \mathrm{kV} / \mathrm{cm}$ elsewhere. In the oil, the goal is approximately $20 \mathrm{kV} / \mathrm{cm}$.

A drawing showing both the triode region and the insulator is shown in Figure 6.

\section{REFERENCES}

1. P.B. Wilson, "RF power sources for 5-15 TeV linear colliders," Intern. workshop on pulsed RF sources for linear colliders, April 1996.

2. S. Humphries, www.fieldp.com, 2003

\section{ACKNOWLEDGEMENT}

This program is funded by the U.S. Department of Energy Small Business Innovation Research Grant number DE-FG03-01ER83209.

Figure 6. Cut-away solid model of the sheet beam gun. 